\title{
Tropisme placentaire du Plasmodium et acide hyaluronique
}

On connaît depuis longtemps la susceptibilité particulière des femmes enceintes au paludisme à Plasmodium falciparum. L'infection, grave chez les primipares, chez lesquelles elle peut entraîner un accouchement prématuré ou une mortalité périnatale, s'atténue au cours des grossesses suivantes. L'adhérence des érythrocytes infectés puis leur séquestration dans les espaces intervillositaires de la face maternelle du placenta avaient suggéré l'existence, sur les syncytiotrophoblastes, de récepteurs fixant sélectivement ces globules rouges. On connaît l'antigène CD36, la molécule ICAM-1 (intercellular cell adhesion molecule) incriminés dans l'adhérence du parasite à l'endothélium, et en 1996, la responsabilité de la chondroïtine sulfate A dans l'adhérence aux syncitiotrophoblastes $\left(\mathrm{m} / \mathrm{s} 1996, n^{\circ} 10\right.$, p. 1178) a été mise en évidence [1]. C'est au tour de l'acide hyaluronique d'être mis sur la sellette par des chercheurs de l'Université de Victoria (Australie), en collaboration avec l'école de médecine tropicale de Liverpool (GB), et l'Université de Blantyre (Malawi) [2]. L'acide hyaluronique, comme la chondroïtine sulfate A, favoriserait l'adhérence des globules rouges infectés par le plasmodium au niveau placentaire, alors que ni le CD36, ni ICAM-1 n'interviennent.

L'acide hyaluronique est un glycosaminoglycane non sulfaté constitué uniquement de disaccharides (un acide hexuronique, et un sucre aminé, la $\mathrm{N}$-acétylglucosamine), assemblés en chaînes parfois très longues pouvant atteindre, sous forme multimérique, plusieurs microns. Il n'est généralement pas couplé à une structure protéique de type protéoglycane, mais reste associé aux protéines de la matrice extracellulaire ou de l'environnement péricellulaire par des liaisons non covalentes. On le trouve en abondance dans le placenta, le cordon ombilical (gelée de Wharton), le corps vitré de l'œil et le fluide synovial. Ses multiples chaînes sucrées lui permettent de couvrir une large surface accessible à l'extérieur de la cellule, et en font évidemment un récepteur idéal. Pour tester son rôle dans le piégeage des globules rouges infectés, les auteurs ont comparé, ex vivo, l'adhérence de globules rouges issus du placenta ou du sang périphérique de femmes enceintes à l'acide hyaluronique purifié. Dans tous ces tests, le résultat est exprimé en nombre de globules rouges par $\mathrm{mm}^{2}$ de surface recouverte du substrat à tester après 30 minutes d'adhérence. Plus de $80 \%$ des échantillons de globules rouges placentaires adhèrent à l'acide hyaluronique, et seulement $50 \%$ des échantillons isolés du sang périphérique, mais le nombre de globules rouges placentaires adhérents est extrêmement variable (de 1 à $1200 / \mathrm{mm}^{2}$ !). Afin de mimer au plus près la situation physiologique, et de prendre en compte les phénomènes rhéologiques, paramètre essentiel de la séquestration des globules rouges infectés au niveau placentaire, ce que ne fait pas le test in vitro, les auteurs ont analysé l'adhérence des globules rouges dans un système de flux, dans lequel la vitesse de flux et les taux de cisaillement peuvent être modulés de façon à reproduire les paramètres rhéologiques caractérisant la circulation dans les vaisseaux placentaires, où la vitesse de flux est lente et les forces de cisaillement faibles. Dans ces conditions, l'adhérence à l'acide hyaluronique des globules rouges infectés, identique à celle observée lorsque la chondroïtine sulfate A est utilisée comme substrat, est importante. In vivo, elle est probablement renforcée par la multiplicité des sites de liaison. In vivo, on peut aussi penser que les globules rouges subissent entre les villosités un processus de "roulement» (rolling) proche de celui qui caractérise la première étape d'adhérence des leucocytes aux cellules endothéliales [3]. Dans le cas de l'acide hyaluronique comme de la chondroïtine sulfate A, molécules ayant une charge anionique très forte, il était important d'éliminer une adhérence ne faisant intervenir que des liaisons de charge et non pas un couple ligand-récepteur. L'absence d'adhérence à l'héparine, de même charge négative, ou après dégradation de l'acide hyaluronique par des enzymes spécifiques, suggèrent que cette fixation utilise un récepteur spécifique. Il est probablement différent de celui qu'utilise le parasite pour se lier à la chondroïtine, puisque la trypsine abolit la liaison à l'acide hyaluronique, mais pas à la chondroïtine. De plus, selon les expériences, les globules rouges se fixent à l'un ou à l'autre substrat, plus rarement aux deux.

Se pose alors le problème de la nature du récepteur de l'acide hyaluronique? On pense aux membres de la famille génique var, présents sur presque tous les chromosomes du Plasmodium falciparum (m/s 1997, $\left.n^{\circ} 6-7, p .858\right)$ et dont on connaît la fréquence des commutations spontanées, jusqu'à $2 \%$ par génération $\left(m / s ~ 1999, n^{\circ} 2, p .598\right)$. Un des produits des gènes var est la protéine de membrane PfEMP1 de $P$. falciparum [4], qui se lie à CD36 et ICAM1. Dans le cas de la chondroïtine, $P$. Buffet et ses collègues de l'Institut Pasteur (Paris, France) ont identifié un autre domaine du gène var (FCR3.var)

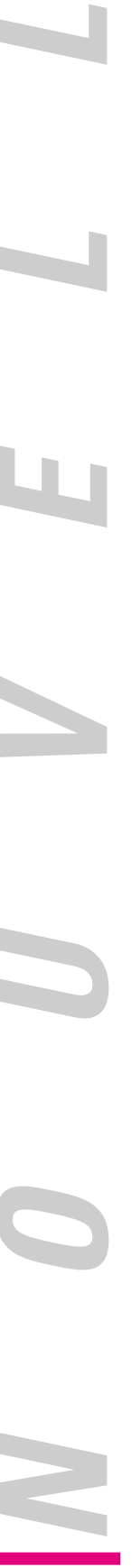


qui code pour une protéine de la famille PfEMP1 exprimée par les globules rouges, sensible à la trypsine, et différente de celle qui se lie au CD36 $[5,6]$. Il est probable que les variations antigéniques de PfEMP1 modulent le tropisme tissulaire du parasite. Quant à la gravité particulière du paludisme de la première grossesse, elle s'explique peut-être par l'absence d'anticorps contre les antigènes du parasite utilisés pour l'adhérence des globules rouges aux syncitiotrophoblastes, alors qu'une immunité s'est déjà développée contre les antigènes utilisés pour l'adhérence à l'endothélium ou à d'autres sites de séquestration. La prophylaxie de la maladie chez les femmes enceintes repose actuellement uniquement sur la chimiothérapie, mais la résistance fréquente aux drogues rendrait fort utile le développement d'autres approches thérapeutiques, dont fait partie l'approche vaccinale.

1. Fried M, Duffy P. Adherence of Plasmodium falciparum to chondroitin sulfate $\mathrm{A}$ in the human placenta. Science 1996 ; 272 : 1502-4.

2. Beeson JG, Rogerson SJ, Cooke BM, et al. Adhesion of Plasmodium falciparum-infected erythrocytes to hyaluronic acid in placental malaria. Nat Med 2000 ; 6 : 86-90.

3. Foussat A, Galanaud P, Emilie D. Les chimiokines et la longue marche des leucocytes. Med Sci $2000 ; 16: 757-66$.

4. Chen BQ, Barragan A, Fernandez V, et al. Identification of Plasmodium falciparum erythrocyte membrane protein 1 (PfEMP1) as the rosetting ligand of the malaria parasite P. falciparum. J Exp Med $1998 ; 187$ : 15-23.

5. Reeder JC, Cowman AF, Davern KM et al. The adhesion of Plasmodium falciparum-infected erythrocytes to chondroitin sulfate A is mediated by $P$. falciparum erythrocyte membrane protein 1 . Proc Natl Acad Sci USA 1999 ; 96 : 5198-202.

6. Buffet P, Gamain B, Scheidig C, et al. Plasmodium falciparum domain mediating adhesion to chondroitin sulfate A: a receptor for human placental infection. Proc Natl Acad Sci USA 1999 ; 96 : 12743-8.

\section{Dominique Labie}

Inserm U. 129, CHU Cochin, 24, rue du Faubourg-Saint-Jacques, 75674 Paris Cedex 14, France. 